\title{
Margaret Fountaine (1862 - 1940). Skizzen aus dem abenteuerlichen Leben einer viktorianischen Schmetterlingsjägerin
}

\author{
Mit 2 Figuren \\ JÜRGEN ECKL ${ }^{1}$ \\ ${ }^{1}$ Hölderlinstr. 9, 65549 Limburg, Germany. - juergen.eckl@googlemail.com \\ Published on 2013-12-20
}

\section{Zusammenfassung}

Biographisches Essay über die britische Entomologin Margaret Fountaine auf Grundlage ihrer tagebuchförmigen Notate, die erst 38 Jahre nach ihrem Tod auf Trinidad im Jahre 1940 der Öffentlichkeit zugänglich wurden. Ihre Herkunft aus der nordostenglischen Provinz in einem viktorianischen Pastorenhaushalt ließen nicht unbedingt erwarten, dass aus einer Liebhaberei eine lebenslange Berufung zur Entomologie werden sollte. Sie erfuhr Förderung durch den Großsammler Henry John Elwes. Durch Erbschaften finanziell einigermaßen unabhängig, unternimmt sie ausgedehnte Exkursionen in Südeuropa, dem Nahen Osten und Nordafrika, schließlich ausgedehnte Sammlungsreisen in alle Erdteile im Auftrag vor allem britischer und amerikanischer Museen und Sammler. Der syrische Christ Khalil Neimy, 15 Jahre jünger als sie, wurde $1901 \mathrm{ihr}$ ständiger Begleiter als Schmetterlingsfänger und bis zu seinem frühen Tod 1929 auch ihr Lebensgefährte. Sie war seit 1898 Mitglied der Royal Entomological Society (ggr. 1833) und wurde 1912 in die Linnean Society of London (ggr. 1788) aufgenommen. Nach ihrem Tod hinterließ sie dem Schloßmuseum von Norwich ihre eigene Sammlung von 22.000 Tagfaltern.

\section{Schlüsselwörter}

britischen Entomologin, Pastorentochter, Weltreisende, Forschungsergebnisse, Vermächtnis

\section{Summary}

Biographicalk scetch on the british entomologis Margaret Fountaine on base of excerpts of her diaries, which not before 38 years after her death in Trinidad 1940 were partially published. Descended from a pastoral household in the North Eastern Anglian province it was not perspective to convert a hobby into a lifetime compassion for entomology. She was promoted by the notorious. Collector Henry John Elwes. Due to heritage she was financially independent and capable to organize extensive excursions in Southern Europe, Middle East and North Africa, finally extensive collecting journeys in all continents in order of British and American museums and collectors. The Syrian Christ Khalil Neimy, fifteen years younger than the lady, became 1901 her permanent companion as butterfly hunter and her lover till his premature death in 1929. Since 1898 she was Member of the Royal Entomological Society (founded 1833) and was in the year 1912 invited to Membership of the Linnean Society of London (founded in 1788). After her death she bequeathed to the Castle Museum at Norwich a collection of 22.000 Diurnal Lepidoptera.

\section{Key words}

British Entomologist, Victorian Heritance, World Wide Expeditions, Entomological results, Legacy 


\section{Einleitung: Ein Leben im Bann der Schmetter- linge}

Der Blick richtet sich auf MARGaret Fountaine, eine große Entomologin um die Wende vom 19. zum 20. Jahrhundert. Zu berichten ist von dem ganz den Schmetterlingen gewidmeten Leben dieser faszinierenden Frau, leidenschaftlichen und überaus erfolgreichen Schmetterlingsjägerin, unermüdlichen Weltreisenden, großartigen Sammlerin und Züchterin, einer wagemutigen und furchtlosen Abenteurerin, einer viktorianischen Lady, praktisch und künstlerisch begabt, voll hochemanzipativer Lebenslust.

MARgARET Fountaine war ausreichend selbstbewusst und sich zeitlebens auch ihrer Anziehungskraft durchaus gewiss, um in den doch sehr männerbestimmten, exklusiven Gesellschaften und Zirkeln britischer und anderer Entomologen Anerkennung und Respekt zu genießen, ähnliches galt für das Aufeinandertreffen mit sardischen Banditen, osmanischen Militärbehörden, österreichischen Zollbeamten, jordanischen Karawanenführern, französischen Kolonialoffizieren, Bürokraten in beiden Amerikas, in Afrika, Asien und Australien, italienischen Verehrern und ungarischen Anbetern.

Zugleich verbarg sie ihr reiches Intim- und Gefühlsleben, aber auch ihre wissenschaftlichen Erkenntnisse und detailgenauen Reiseschilderungen in zwölf telefonbuchdicken Folianten, ihren sogenannten Jahrbüchern in Tagebuchform, im eleganten und ironischen Stil ihres literarischen Vorbildes JANE AUSTEN geschrieben, vor ihrer eigenen Umwelt. Testamentarisch hatte sie 1939 kurz vor ihrem Tod verfügt, dass dieses Vermächtnis für weitere fast vier Jahrzehnte in einem versiegelten Blechkoffer im Castle Museum von Norwich, Norfolk, GB verwahrt und erst ab dem 15. April 1978 der Nachwelt zugänglich gemacht werden durfte. ${ }^{1}$

\section{Die Weltlust der ältesten Tochter eines Land- pastors}

Margaret Elizabeth Fountaine wurde am 16. Mai 1862 als zweites Kind und die älteste Tochter des Reverend John Fountaine, Pfarrer von South Acre, ein Dorf

\footnotetext{
${ }^{1}$ Das gesamte handschriftliche Notat im Umfang von mehr als 3000 Seiten, mit unzähligen Fotografien, Zeichnungen und Einfügungen ist bis heute erst zum geringsten Teil ausgewertet und publiziert. Das Verdienst einer ersten Herausgabe von Auszügen kommt dem Journalisten der Sunday Times. H.W. Cater zu Gute, dessen Augenmerk sich jedoch vorrangig auf die Entwicklung der imponierenden Persönlichkeit von Margaret Fountaine richtet, ihre Reiseabenteuer und Liebesbeziehungen, während die Entomologie das eher exotische Rahmenwerk abgibt.
}

30 Kilometer von Norwich entfernt, geboren. Sie hatte sieben Geschwister, zwei Brüder und fünf Schwestern. Die Pfarrersfamilie selbst war vergleichsweise gering begütert, aber reiche Erbonkel und -tanten im näheren Verwandtschaftskreis verhießen eine bessere pekuniäre Zukunft. Der Vater war ein passionierter Jäger und Sportler, was auf Kindheit und Charakter seiner Ältesten reichlich abfärbte, während MARGARETs Verhältnis zur strengen und gefühlskalten Mutter zu deren Lebzeiten stets äußerst unterkühlt blieb. Kurz nach dem Tod des Vaters im Dezember 1877 übersiedelt die Familie nach Norwich. Bei ihrer Ankunft in dem Stadthaus Eaton Grange beginnt MARgareT mit ihrem ersten Jahrbuch mit dem Datum 15. April 1878 (ihrem wohl fiktiven Geburtstag), das mit allen Folgenden erst genau einhundert Jahre später einer kleinen Öffentlichkeit zugänglich werden sollte. Ihre Tagebücher wiederspiegeln viel jugendliche Liebespein, aber keinerlei viktorianischer Enge, Mit Zwanzig verliebt sie sich unsterblich in SEPTIMus Hewson, ${ }^{2}$ einen irischen Sänger im Kirchenchor und Hallodri. Die ,größte Liebe ihres Lebens' scheitert endgültig nach vier Jahren durch dessen Flucht nach Irland. Zu Margarets Glück verbindet sich dies mit dem Ableben eines ihrer Erbonkel, womit sie mit vierundzwanzig Jahren endlich dank einem auskömmlichen Jahresdeputat ihre weitere Zukunft in Unabhängigkeit planen kann. So hat sich Septimus Hewson um die Entomologie dank seiner Treulosigkeit verdient gemacht, denn sonst wäre Miss Fountaine ihrer eigentlichen Bestimmung, nämlich der Schmetterlingsforschung verlustig gegangen.

So lässt sie auch die Jahre der zeichnerischen Beschäftigung mit den Innenräumen von Kathedralen in Ostengland hinter sich, bereist Europa oft zusammen mit einer ihrer vielen Schwestern und widmet sich ihrer neuen, plötzlich aufgetretenen Leidenschaft: dem Jagen und Sammeln von Schmetterlingen, zuerst in Südfrankreich und der Schweiz, wenig später im Jahr 1893 schon auf Korsika.

„Bevor RACHEL und ich zu dieser Reise aufgebrochen waren, hatte der größte Teil unserer Freunde und Bekannten die faszinierende Seite dieses Unternehmens vollkommen ignoriert - die Schmetterlinge nämlich, von denen es manche Arten sonst nirgends auf der Welt gibt und uns als zwei ziemlich abenteuerlustige und närrische Personen, die sich der Gewalt korsischer Banditen auslieferten, betrachtet. “3 Wie fast erwünscht, trifft Miss FounTAINE in der Einsamkeit der korsischen Berge mehrmals mit dem damals berühmtesten Banditen, JACQUEs BELLACOSCIA, zusammen, der wegen einer Vielzahl von

\footnotetext{
2 „Die größte Leidenschaft und vielleicht hochherzigste Liebe meines Lebens war ohne Zweifel Septimus Hewson, und die Wunde, die er mir durch sein rücksichtloses Benehmen zufügte, hinterließ eine Narbe, die auch nach so vielen Jahren nie ganz zu heilen vermochte." Fountaine, Margaret: (1983) Ich sammle nicht nur Schmetterlinge... Reisen und Abenteuer einer viktorianischen Lady, Wien, Hamburg, S. 50.

${ }^{3}$ Ibid. S. 69 f.
} 
Morden „in Selbstverteidigung“ bereits fünfundzwanzig Jahre im Maquis unterwegs war. Unsere Heldin aber vermerkt lakonisch: „Ich trank gemeinsam mit JACQUES, und in manchen Zeiten, wenn mein Leben in ruhigeren Bahnen verläuft, denke ich gern an jene wilde Berggegend zurück, an den Verbrecher und seine Sippschaft, an die scharfen Hunde; die zwischen den grauen Felsen und im violetten Heidekraut umherstreifen...das ist ein starker Kontrast zur langweiligen Friedlichkeit eines englischen Heims. “4 Margaret Fountaine war damit in der Entomologie und ihren damit verbunden eigenartigen Reizen angekommen.

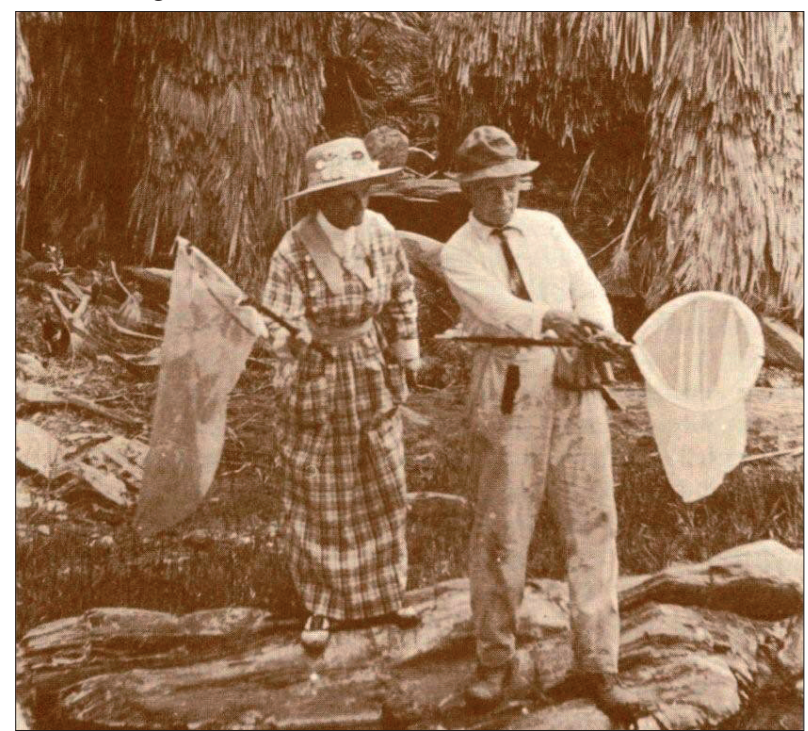

Fig. 2: Auf Schmetterlingsjagd in Costa Rica. Margaret Fountaine mit Khalil Neimy 1911.

Was Margaret Fountaine in den Anfängen ihrer entomologischen Arbeit noch fehlte, war ein strukturierter und systematisch begründeter Zugriff auf ihr künftiges Lebensthema. Hier erhielt sie den entscheidenden Anstoß durch die Begegnung mit dem weitgereisten Großsammler, Botaniker und Entomologen Henry John Elwes (1846-1922), die ihr Deputatverwalter und Erbonkel JoHn LAwEs 1895 vermittelt hatte. Anfangs löste dieses Zusammentreffen bei ihr eine mittlere Depression aus: „Ich war so lange glücklich, bis RACHEL und ich für drei Tage nach Colesborne eingeladen wurden, um uns Mr. Elwes' Schmetterlinge anzusehen, was ich mir ganz besonders gewünscht hatte...Nach dem Luxus und der Vornehmheit eines privaten Landsitzes, nach dem Aufenthalt in Gesellschaft von Menschen meines eigenen Standes und nicht zuletzt nach der Besichtigung der wunderbaren Sammlung von Mr. Elwes, die einen ganz unsicher und unzufrieden macht, hasste ich die Gewöhnlichkeit der Leute in der Pension und war mit meiner eigenen kleinen Sammlung so unzufrieden, das es fast schon kindisch war. ,Nun können sie sich eine Vorstellung von den Möglichkeiten des Schmetterlingsammelns machen', hatte Mr. Elwes eines Tages im Ausstellungsraum zu mir gesagt, worauf ich erwidert

${ }^{4}$ Ibid. S. 72 hatte, dass ich im Gegenteil nur die Unmöglichkeit des Sammelns erkannt hätte. “5

Die Koketterie mit ihrem Unvermögen war von kurzer Dauer, vielmehr wurde ihr Ehrgeiz durch die Begegnung mit ELwEs erst richtig angestachelt, vor allem befolgte sie seinen Rat, sich bei Sammelexkursionen auf die Suche nach schwer erreichbaren, selteneren Arten zu konzentrieren, alle anderen stellten sich dann schon ganz nebenbei ein. Entsprechend richtete Margaret Fountaine bei ihrer Sizilienreise im Folgejahr ihr Augenmerk auf die sehr lokale und endemische Art des Schachbrettfalters

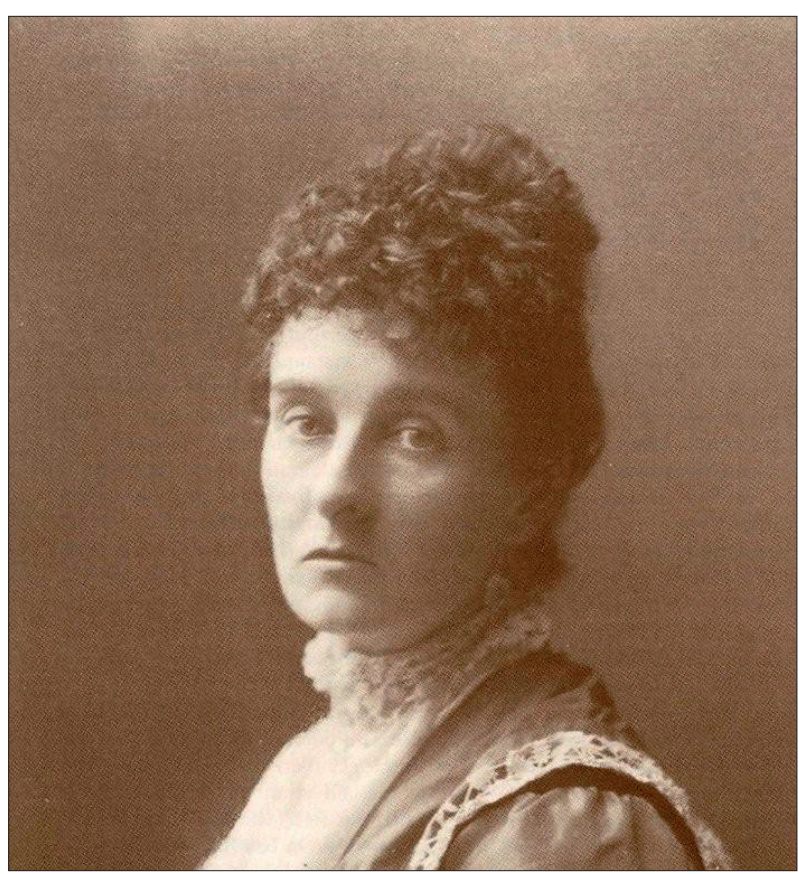

Fig. 1: Margaret Fountaine.

Melanargia pherusa und war damit sehr erfolgreich ${ }^{6}$, auch wenn sie bei jedem Aufbruch zur Jagd noch in Palermo feststellen musste: „Ich würde allerdings übertreiben, wenn ich behaupten wollte, dass mir die Aufmerksamkeit, die ich erregte, wenn ich in meiner Schmetterlingsausrüstung - Netz, Rucksack, alles komplett - den Corso entlangging, auch nur im mindesten angenehm gewesen wäre...Wenn ich dann zu anderen Zeiten äußerst fein gekleidet denselben Corso entlangging, um einzukaufen oder sonst etwas zu tun, konnte ich allerdings nicht begreifen, wie ich es überhaupt je wagte, mich mit der

\footnotetext{
${ }^{5}$ Ibid. S. 83.

6 „Ich fuhr also nach Bocca di Falco, ein langgestrecktes, schmutziges Dorf voller Hühner und Ziegen. Dort jagte ich viele Stunden jener herrlichen Sommertage den Pherusa, ein wildes, windgetriebenes Geschöpf, das mich zu einer langen und mühseligen Jagd über lockeres Geröll und dichtes Gestrüpp verleitete, nur um mir am Ende zu entwischen. Aber dieser Schmetterling trat hier so häufig auf, dass ich sogleich einen anderen sah, wenn ich den einen aus den Augen verloren hatte, und deshalb immer mit voller Botanisiertrommel nach Hause kam. "Ibid. S. $84 \mathrm{f}$
} 
Schmetterlingsausrüstung unter diesen vielen eleganten Leuten zu zeigen. ${ }^{\text {7 }}$

Solche widerstrebende Erfahrungen führten oft dazu, dass Entomologen immer schon gern Vereine bildeten. Margaret Fountaine sollte dies im Jahre 1898 bei einer Reise nach Budapest aufs Beste erfahren: „Schließlich ging mir ein Licht auf: Ich war beim wöchentlichen Treffen der Budapester Entomologischen Gesellschaft... Männer aller Altersgruppen waren anwesend, von zwanzig bis sechzig, und eine lustigere und glücklichere Gesellschaft hätte ich kaum finden können... Natürlich war ich die einzige Frau unter den Anwesenden, weshalb sie sich auch nicht genug um mich bemühen konnten...Der Wein floß reichlich, und die Scherze nahmen kein Ende, obwohl ich, da sie ständig Ungarisch sprachen, nicht das geringste verstand. Aber es machte mir trotz allem großen Spaß. ${ }^{\text {8 }}$ Nach gemeinsamen Exkursionen und intensiver Sammeltätigkeit ging der Aufenthalt mit einem weiteren Höhepunkt zu Ende: „Zurück in Budapest verabschiedete ich mich am Vorabend meiner Abreise beim Entomologen-Treffen mit einer Rede. Da der Vorsitzende darauf bestanden hatte, mich reichlich mit Wein zu versorgen, um meiner Beredsamkeit nachzuhelfen, brachte ich sie, wie ich glaube, recht gut hinter mich, vor allem, wenn man bedachte, dass jedes Wort in Deutsch sein musste. Jedenfalls dankte man mir mit lautem Applaus und Gläserklirren. “9 Nach ihrer Rückkehr nach England wurde sie noch im gleichen Jahr 1898 Mitglied der Royal Entomological Society (gegründet 1833).

Im Sommer 1900 ist Margaret Fountaine zu einer gemeinsamen Schmetterlingsexpedition in Griechenland mit ihren alten Lehrmeister HENRY JOHN ELwEs verabredet, von dem sie mit sanfter Ironie berichtet: „Mit Mr. Elwes zu reisen brachte ziemliche Bequemlichkeiten mit sich. Niemals machte er sich die Mühe, irgend etwas zu tun, was sein Führer für ihn erledigen konnte. Niemals aß er minderwertige Nahrungsmittel, wenn die Möglichkeit bestand, sich bessere zu verschaffen, und er ging niemals zu Fuß, wenn ein Pferd oder ein Maultier verfügbar war. Dies alles kam auch mir zunutze. Unglücklicherweise musste er dann ganz plötzlich nach England zurück. ${ }^{10}$

MARgaret Fountaine wird dies plötzliche Abreise eher für Elwes, denn für sich als unglücklich eingeschätzt haben, war sie doch eine begeisterte Fußgängerin und liebte es, weite Strecken über Berg und Tal mit dem Fahrrad zu bewältigen, wie sie es auf ihren Exkursionen in Frankreich, der Schweiz, Italien und Ungarn mit phänomenalen Tagesmärschen von dreißig und mehr Kilometern unter Beweis gestellt hatte. Die neue Situation in Griechenland gab der 38-Jährigen dafür gute Gele-

\footnotetext{
${ }^{7}$ Ibid. S. 85 f.

${ }^{8}$ Ibid. S. $114 \mathrm{f}$.

${ }^{9}$ Ibid. S. 119.

${ }^{10}$ Ibid. S. 128 .
}

genheit, etwas von ihrer Lebensphilosophie in ihr Tagebuch zu notieren: „Zwei Nächte verbrachte ich in Athen, sehnte mich aber in die Wildnis zurück, wo ich mein ungezwungenes Leben führen konnte, fernab von allen Konventionen der Zivilisation. Freiheit ist das größte Glück meines Lebens. Gott sei Dank gibt es wenige Menschen, die mir etwas bedeuten. Ich wollte, es gäbe überhaupt niemanden. Bevor ich diese schöne Welt verlassen muss, möchte ich noch so viel wie möglich von ihr sehen, und das Leben ist so entsetzlich kurz. Gefühle hindern uns daran, Großes zu unternehmen, Gefühle fesseln uns an einen einzigen Ort -wenn nicht in räumlicher, so doch in geistiger Hinsicht. Und wenn dann alles zu Ende geht, ist das Leben vorbei, und wir haben nichts von all den großen Dingen verwirklicht, die wir in unseren begeisterten Träumen anstreben." ${ }^{11}$

Margaret Fountaine konnte in ihrem langen Leben ihre Träume einholen, aber einiger Gefühlswirrwarr blieb ihr zum Glück nicht erspart.

\section{Margaret Fountaines Lebensgefährte KhaliL "ChARLES" NeIMY}

Nach einem traurigen Winter in England und der Regelung einiger Finanzfragen bezüglich ihres Deputats reiste Margaret Fountaine im Mai 1901 über Beirut nach Damaskus, um den Vorderen Orient lepidopterologisch ausgiebig zu bearbeiten. Gleich nach ihrer Ankunft heuerte sie einen jungen sogenannten ,Dragoman', einen Dolmetscher und Reiseführer an, der sich ihr als syrischer Christ griechischer Herkunft, geboren in Kairo und sein eigenwilliges Englisch mit starkem amerikanischen Akzent als Ergebnis von vier Schuljahren in Wisconsin vorstellte. Schon bald wurde der 39-Jährigen englischen Lady deutlich, dass sich ihr fünfzehn Jahre jüngere Führer und Helfer in sie rettungslos verliebt hatte. MARGARET verhielt sich aus-, aber nicht unbedingt abweisend, wich aber erst einmal nach Beirut zurück, um sich dort mit Henry John Elwes zwecks entomologischer Exploration des Libanon zu treffen. Dieser hatte aber bereits eine Nachricht hinterlegt, dass er von der Exkursion absehen müsse, weil laut Times über Beirut eine Quarantäne verhängt worden sei. Zugleich traf von Khalil Neimy ein Telegramm ein, dass er mit ihrem Lohnangebot für eine Daueranstellung einverstanden sei und unverzüglich anreisen werde. Was dem Wiedersehen folgte, bleibt in den Tagebuchaufzeichnungen unklar, aber das Hin und Her der Gefühle gipfelte in einer sehr romantischen Szene: „Schuldbewusst dachte ich daran, wie jung er war, und gestand ihm mein wahres Alter. Ich sagte ihm, dass ich gerade mein neununddreißigstes Lebensjahr vollen-

${ }^{11}$ Ibid. 
det hätte, und dachte bei mir, dass ihn dies schließlich davon abbringen würde - er war knapp vierundzwanzig. Aber ich hatte mich geirrt. Und dann versprach ich an diesem herrlichen Sommermorgen dort draußen im Schatten der riesigen Felsen in der Nähe Baalbeks KHALIL Neimy feierlich, seine Frau zu werden. Und ich sagte: ,Ich habe dich nicht ein einziges Mal geküsst, aber jetzt werde ich dir zum ersten Mal einen Kuss geben' - und ich küsste ihn auf die Wange, die weich und rosig wie die eines kleinen Jungen war. Dann fassten wir einander bei den Händen und gelobten, einander die Treue zu halten. Während dieser ganzen Zeit flatterten die großen braunen Schmetterlinge ungestört zwischen den heißen Felsen hin und her." 12

Trotz des chaotischen und leicht verklemmten Beginns hielt diese später sehr innige Liebesbeziehung der beiden die folgenden siebenundzwanzig Jahre bis KHALIL ,CHARles' im Juni 1929 im fernen Damaskus an den Spätfolgen einer Malariainfektion starb, die er sich zusammen mit ihr 1904 in Algerien zugezogen hatte, die aber bei Margaret ohne dauernde schwere Nachwirkungen geblieben war. Trotz langer Trennungen aus Geldmangel und gelegentlich logistischen Gründen hielt diese Lebenspartnerschaft, obwohl nie eine formelle Ehe zu Stande kam, ungeachtet der Anstrengungen KHALILs seine verzwickten Staatsbürgerschaften, Religionszugehörigkeiten und Familienstände zu ordnen. Beide pflegten in ihrem Verhältnis eine durchaus postviktorianische Toleranz, ab und zu nur gefährdet durch periodische auftretenden Eifersuchtsanfälle Khalils und seiner Neigung $\mathrm{zu}$ exzessiven Alkoholgenuss. Er selbst entwickelte zu den Schmetterlingen ein von seiner Partnerin geprägtes Engagiertsein und erreichte bei Fang und Präparation vergleichbare Fähigkeiten und Geschicklichkeit wie seine Frau. Die gemeinsam zusammengetragene Sammlung wuchs derart, so dass MARgaret Fountaine 1908 in der Sheriff Road, West Hamstead im Nordwesten Londons ein Studio anmietete, um ihre Schätze angemessen aufzubewahren und zu bearbeiten, gesichert auch während ihrer langen Reisen. Bei den verschiedenen kurzen Aufenthalten auf der Insel nahmen beide gelegentlich an großen wissenschaftlichen Entomologentreffen teil, bei der sie dankbar die wachsende Anerkennung in der britischen Entomologengesellschaft genoss, die auch ihren Lebensgefährten mit einbezog.

Ein Höhepunkt war dabei anlässlich des Second International Congress of Entomology 1912 in Oxford die Einladung an Margaret Fountaine durch den damaligen Präsidenten der Linnean Society of London, EDWARD Baguali Poulton, Mitglied dieser ehrwürdigen und ältesten - gegründet 1788 - naturforschenden Gesellschaft der Welt zu werden. ${ }^{13}$

\footnotetext{
${ }^{12}$ Ibid. S. 137 f.

${ }^{13}$ Ibid. S. 208.
}

Ihre Sammeltätigkeit weitete sich immer stärker auf tropische Zonen aus, nur während des Ersten Weltkriegs unterbrochen durch ihren drei Jahre dauernden verzweifelten Versuch, im australischen Queensland eine Pferdefarm aufzubauen. Das Projekt scheiterte grandios an Australiens heftiger Natur, fehlendem Geld, australischen Nachbarn und wirtschaftlichem Unvermögen der beiden Schmetterlingssammler. ${ }^{14}$

Nachdem das Anlagedeputat von Margaret Fountaine während des Weltkrieges in britische Kriegs(zwangs) anleihen umgewandelt worden war, standen beide ohne finanzielle Mittel da. Während KhaliL NeImy als Kleinkrämer in Sydney blieb, um aus ihren Hinterlassenschaften - wenn möglich - noch einen Erlös zu ziehen, durchquerte MARgaret Fountaine zum Jahreswechsel 1917 den Pazifik mit Ziel Los Angeles und kam dort als erstes bei einer Dependance des französischen Roten Kreuzes unter, um drei Tage in der Woche Bandagen für Kriegsverletzte zu wickeln, den Rest der Woche streifte sie durch die Berge Hollywoods auf der Suche nach interessanten Schmetterlingen. Später arbeitete sie in einem Andenkenladen in Pasadena für einen Vierteldollar die Stunde, sammelte für ein Institut Spinnennester für vier Dollar das Dutzend und nahm dann unter dem Stichwort, Butterfly Harvest' eine Bestellung für 5000 Exemplare des Pygmäenbläulings Brephidium exilis für einen Schmuckhändler in Pasadena an. ${ }^{15}$

Als sie einiges gespart hatte, leistete sie sich im Dezember 1919 ein Rundfahrtticket von San Francisco nach Wellington und zurück von Auckland nach Vancouver mit einem Jahr Gültigkeitsdauer, um in Neuseeland endlich ihren geliebten KHALIL wieder zu treffen, dessen Gesundheitszustand durch mehrere Malariaschübe sehr angegriffen war. Das Wiedersehen wurde getrübt durch KHALILs dringendes Verlangen, seine alte Mutter, die vielleicht im Sterben lag, in Damaskus sehen zu müssen. So fingen beide zwar ausreichend Exemplare des Roten Admirals Vanessa gonerilla, die silberstreifige Satyride Agyrophenga antipodum und den dunkelvioletten Feuerfalter Lycaena boldenarum und damit fast ein Viertel der Tagfalterfauna Neuseelands, aber das Gefühl wechselseitiger Entfremdung nahm überhand. Im Dezember 1920 trennten sie sich: KHALIL Richtung Port Said und dann nach Damaskus, Margaret Richtung San Francisco und dann Chikago. Erst die anstrengende Sammelreise nach Burma und Hinterindien 1922 brachte die Liebenden wieder zusammen.

\footnotetext{
${ }^{14}$ Die Pleite ihres ,australischen Exils' war dergestalt deprimierend, dass ihre Schilderung erst im Jahr/Tagebuch 1921 nachgeholt wurde. Auszüge in Fountaine, Margaret (1987): Butterflies \& Late Loves, S. 21-41.

${ }^{15}$ Ibid. S. 50: „I got my 5,000 Exilis, and refused to undertake the other 5,000, though much urged by Mr. NewCOMB to do so."
} 


\section{Schmetterlingssammeln als Brotberuf}

Bei ihrer ausgedehnten Reise auf die Philippinen beim Fang der bei den britischen Sammlern hochbegehrten wunderschönen Papilioniden, vor allem des wie ein Opal leuchtenden Vogelfalters Troides magellanus kam erstmals der wirtschaftliche Faktor der Entomologie bei Margaret Fountaine und ihrem Lebensgefährten zum Tragen. Zwar nicht als Schmetterlingshändler wie das Dresdner Haus Staudinger, aber als Auftragsnehmerin der britischen Großsammler wie Lord Walter Rothschild ${ }^{16}$, des alten Elwes, des ehrgeizigen Longsdon; aber auch amerikanische Entomologen, die sie auf ihren Streifzügen in den USA kennengelernt hatte, waren ihre Kunden. Zur Gute kamen ihr dabei auch ihre Erfolge als Züchterin tropischer Falter, was neben dem Aufspüren und Fangen auch während ihrer Sammelreisen mehr und mehr ihre Hauptbeschäftigung geworden war.

So suchte sie in Kommission für den Meistersammler DAvid LoNGSDon, der stolz darauf war, keinen einzigen Falter seiner riesigen Sammlung selbst gefangen zu haben, die verschiedenen Formen des süd- und mittelamerikanischen Schwalbenschwanzes Battus polydamas auf den Inseln der Kleinen Antillen. LongsDon bestand darauf, nur gefangene, keinesfalls gezüchtete Exemplare anzunehmen und schrieb ihr auch die Reiseroute von Insel zu Insel vor. Nach MARgarets Aufzeichnungen war David LONGSDON, eine lunatische Nervensäge, aber, sie musste ihren Job tun. ${ }^{17}$ Und so reiste MARGaret Fountaine von St. Lucia nach Antigua, von dort nach Dominica, dann nach St.Vincent, darauf ins kolonialfranzösische Martinique und das malerische Guadeloupe. Der Job lief gut, sie konnte einiges Material für die eigene Sammlung abgreifen und trotzdem den Auftrag voll erfüllen. Nur hatte sie den Kontakt mit KHALIL währenddessen verloren, der vor dem englischen Winter nach Damaskus geflüchtet war und jetzt noch einige Wochen in Kairo daran gehängt hatte. Es waren fast die letzten Nachrichten ihres Gefährten. Das Fieber kehrte zurück und ließ ihn nicht mehr aus seinen Fängen bis zu seinem frühen Tod im Juni 1929.

Ähnliche Aufträge, die sie gewissenhaft erfüllte, halfen ihr auch nach KHALILs Tod über den Verlust hinweg, da sie so beständig auf Reisen sein konnte und sich auch als Siebzigjährige keineswegs zu schonen gedachte. So verließ sie das traurige London und fuhr nach Rio de Janeiro, um in die brasilianischen Tropenwälder einzutauchen, wo jede Sandbank entlang der Flüsse ein Schmetterlings-

\footnotetext{
${ }^{16}$ Bei einem Besuch in dessen Sammlung im eigenen TringMuseum schlüpfte sie in die Rolle der Königin von Saba vor Salomo und rief wie diese aus: „Und nicht einmal die Hälfte hat man mir berichtet!", was angesichts einer Sammlung von einer Dreiviertel Million Schmetterlingen die Eigene mit damals erst(?) 16.000 Faltern etwas ärmlich erscheinen ließ. Ibid. S. 79

${ }^{17}$ Ibid. S. 93.
}

paradies sein kann. ${ }^{18}$ Statt sechs Wochen blieb sie ganze sechs Monate, hauptsächlich am Amazonas und seinen Zuflüssen. Einen immer größeren Raum nahm nun auch die zeichnerische Darstellung der verschiedenen Stände in der Metamorphose von tropischen Schmetterlingen ein, oftmals die ersten Darstellungen ihrer Entwicklung in der Wissenschaft. Weit über 1000 Blatt werden es am Ende sein, die dank der zeichnerischen Ausbildung der jungen Margaret Fountaine in den Kathedralen von Norfolk von exquisiter Delikatesse und Präzision sind. Sie bereiste Westafrika, Südamerika, auf Trinidad bestieg sie 1931 ihren persönlichen Zauberberg, den Mt. Benedict. Das Studium des Afrikabandes von Adalbert Seitz: Die Großschmetterlinge der Erde, beflügelt sie zu einer groBen Sammelreise nach Madagaskar, mittlerweile 71 Jahre alt. Es folgen Ostafrika, Indochina. Ihre letzte vollendete Reise führt sie kurz vor Kriegsausbruch nach Celebes/ Sulawesi. Nach England zurückgekehrt hinterlegt sie ihr Testament, bevor sie sich zu ihrer letzten Fahrt nach Trinidad einschifft. Am 21. April 1940 wird Margaret Fountaine von Bruder BRUNo aus der Benediktinerabtei des Mt. Benedict auf der den Berg hinauf führenden Straße gefunden, wo sie zusammengebrochen ist. Das Schmetterlingsnetz liegt daneben. Er trägt sie in das Gästehaus der Abtei, wo sie stirbt.

\section{Die Reisen der Margaret Fountaine}

"Über ihr ganzes und extrem unkonventionellen Lebens hinweg wurde Margaret Fountaine vielleicht die meistgereiste britische Entomologin, die je gelebt hat. ${ }^{\text {"19 }}$

Sizilien 1896 - Ungarn/Herkulesbad 1897 - Südfrankreich 1898 - Norditalien 1898

Ungarn /Budapest1898 - Griechenland 1900 - Syrien 1901 - Österreich 1901 - Syrien 1902

Kreta 1902 - Türkei 1903 - Algerien 1904 - Italien 1905 - Spanien 1905 - Korsika 1906 - Bosnien/Herzegowina 1907

Südafrika 1908/1909 - USA/Virginia 1910 - Jamaika 1911 - Costa Rica 1911 - Indien 1912

Ceylon-Sikkim-Tibet 1913 - Penang(Malaya)-SingapurBatavia(Djakarta)-Port Moresby/Neuguinea 1913

\footnotetext{
${ }^{18}$ Ibid. S. 101.

19 „Over her long and extremly unconventional life Margaret Fountaine became perhaps the most travelled British lepidopterist who has ever lived." Michael A. Salmon, Peter Warren, Basil Harley (2000): The Aurelian Legacy: British Butterflies and Their Collectors, Berkeley, S. 200.
} 
Australien 1914-1917 - Kalifornien-Texas-LousianaVirginia 1917 - Arizona-New Mexico-Kalifornien 1918/1919 - Fidschi-Inseln-Neuseeland 1920 - UtahMichigan-New York 1921 - Burma 1922 - ThailandHongkong 1923/1924 - Philippinen 1925

Kanarische Inseln-Westafrika-Nigeria-Guinea 1926 Karibik 1928 - Brasilien 1930

Britisch-Guyana 1931 - Venezuela 1931 - Trinidad 1931 - USA 1932 - Kuba 1932 - Madagaskar 1933 - Uganda 1934 - Uganda-Tanganyika-Kenia 1935-1936

Trinidad 1937 - Indochina 1938 - Celebes 1939 - Trinidad 1940

\section{Das Vermächtnis der MaRgaRET FountaInE}

In ihrem Testament vermacht die Entomologin ihre Sammlung mit mehr als 22.000 hervorragend präparierten und bezettelten Exemplaren von Tagfaltern dem Schlossmuseum von Norwich. Die Sammlung soll im Ganzen erhalten bleiben und für alle Zeit den Namen „The Fountaine-Neimy Collection“ tragen. Dazu gehörte eine versiegelte schwere schwarze Kiste versehen mit dem Warnhinweis: „Not to be opened until April 15, 1978“ unterschrieben und datiert: September 5,1939 .

Norman Riley, Kustos der Entomologie im Britischen Museum wird mit der Überführung der Sammlung aus dem Studio in Westham nach Norwich ins Castle Museum beauftragt und erhält für die Mühe $100 \mathrm{E}$. Ihre Zeichnungen und Skizzenblätter und Sonderdrucke gehen an das Naturkundemuseum in London. Ihre Sammelausrüstungen und Bücher bestimmt sie für das jüngste Mitglied der Royal Entomological Society. Persönliche Habe, Schmuck und Donationen erhalten mehrere Neffen und Nichten.

Die versiegelte Kiste wird am 17. April 1978 geöffnet (der 15. April fiel auf einen Samstag), obenauf liegt ein Brief, dessen letzter Absatz lautet: „To the reader, maybe yet unborn, I leave this record of the wild and fearless life of one of the ,South Acre children' who never grew up und who enjoyed greatly and suffered much. M.E. Fountaine" 20 .

\footnotetext{
${ }^{20}$ [Ich hinterlasse dem Leser, vielleicht einem noch ungeborenen, diesen Bericht über das wilde und furchtlose Leben eines der Kinder aus ,South Acre, das nie erwachsen wurde und das so großen Spaß hatte und viel litt.] M.E. FonTAINe zitiert nach diess. (1987): Butterflies and late Loves, S. 132.
}

\section{Margaret Fountaine im widersprüchlichen Urteil der Nachwelt}

Obwohl Norman Riley (1890-1979) bei seiner ersten Begegnung als junger Captain mit MARgaret FounTAINE im Jahr 1913 von der Attraktivität der damals Fünfzigjährigen äußerst überrascht war ${ }^{21}$, und er als einer der Vollstrecker ihres Testaments alle Aufträge getreulich umgesetzt hatte, äußerte er sich befragt vom Herausgeber ihrer Tagebuchaufzeichnungen W.F. CATER nach ihren Qualitäten als Entomologin in einer Weise, die selbst für den langjährigen Kustos der Entomologie im Britischen Museum sehr überheblich und dünkelhaft daherkam: „Naturalists, particularly the kind who take a pride in building up valuable collections, are notoriously bad at recording their observations for the benefit of others. Miss Fountaine was one such. Her knowledge of the ways of tropical butterflies was profound, probably unique, but only here and there in her diaries and published accounts of her journeys do unexpected records of odd events crop up... She was rather a loner, with few really good friends even among entomologists and this and her uncontrolled wanderlust may account for her recording so little of her work in print or any systematic form. She undoubtedly enjoyed her life of butterfly catching but failed to catch that one deep happiness she ever sought." ${ }^{22}$ Hier verschafft der Museums-Entomologe Norman RiLey seinem Unmut Luft über Leute, die finanziell abgesichert nach Lust und Laune in der ganzen Welt herumreisen und nach eigenem Gutdünken sammeln können, ohne sich groß an die Vorgaben der Systematik zu halten. Sein Urteil über das Lebenswerk der Margaret Fountaine hatte Gewicht wegen RiLeys dominanter Stellung in der britischen Entomologie als Verwalter und Bearbeiter der immensen Sammlungen des Britischen Museums, er galt

\footnotetext{
${ }^{21}$ „Having heard something of Miss Fountaine's exploits the announcement conjured up visions of a well-worn battle axe ... instead I met a tall, attractive, rather frail-looking, diffident but determined middle-aged woman. The strongest impression she gave me was one of great sadness. It was not long, however, before IU discovered that this veil of sadness could be penetrated by self-deprecating flashes of humour that quite transformed her..." zitiert in MARgaret Fountaine (1987): Butterflies and late Loves, S. 134.

${ }^{22}$ Ibid. S. 136 [,Naturforscher', vor allem die Sorte, die stolz sind, eine wertvolle Sammlung aufzubauen, sind bekanntermaßen schlecht darin, ihre Beobachtungen aufzuschreiben zu Gunsten Anderer. Miss Fountaine war eine davon. Ihre Kenntnis der Wege tropischer Schmetterlinge war profund, vielleicht einzigartig, aber nur hier und da in ihren Tagebüchern und veröffentlichen Reiseberichten werden Unerwartetes oder Überraschendes angeschnitten... Sie war eine ziemliche Einzelgängerin mit nur wenigen wirklich guten Freunden auch unter Entomologen, dies und ihre unkontrollierte ,Wanderlust ' mögen verantwortlich dafür sein, dass in ihrem Werk so wenig gedruckt oder in systematischer Form auftaucht. Sie genoss zweifellos ihr Leben beim Schmetterlingsfang, aber es gelang ihr nicht, das tiefe Glück, das sie immer gesucht hat, einzufangen.]
} 
als herausragender Systematiker mit einer ungeheuren Zahl veröffentlichter Spezies-Listen, war aber nur mit bescheidenen Feldforschungserfahrungen ausgestattet. 1970 hatte er gemeinsam mit Lionel Higgins das Standardwerk: A Field Guide to the Butterflies of Britain and Europe in London veröffentlicht. ${ }^{23}$

RiLEYs kaum versteckte Misogynie in seinen nur als Typoskript vorliegenden Äußerungen waren für BARBARA T. GATES in ihrer umfassenden Untersuchung des Beitrags viktorianischer Frauen zur Naturforschung ${ }^{24}$ Anlass dessen Rolle direkt zu sezieren: „In fields like entomology, men like Riley had appropiated what was proper to see and therefore to know this diminishment of FounTAINE's work was not pronounced with an edge of disdain or even disrespect but with the ring of authority. His was the official word, based on official seeing; it was what every self-respecting sientist would propound. Self-taught field naturalists were simply less and less valuable to the scientific enterprise - and, for the most part, women landed in the camp of self-taught and unofficial." ${ }^{25}$

Nicht ganz so ernst geht die Reiseschriftstellerin und Abenteuerin Natascha Scott-Stokes in ihrer Biographie $^{26}$ zu Werke, die in ihr eine Vorläuferin aus viktorianischen Zeiten sieht, wobei die Autorin das Bild das durch die bisherige Auswahl ihrer Tagebuchnotizen entstanden sei, „als einer exzentrischen Viktorianerin mit einer hemmungslosen Leidenschaft für Männer “27 korrigieren möchte, indem sie eher die melodramatische Seite von Fountaines Leben hervorhebt und im weiteren auch die Identifikation der Autorin mit ihrem biographischen Subjekt übertreibt. Sсотт-SтокEs kondensiert die von ihr gewählten Auszüge aus Fontaines Tagebüchern ganz auf die emotionale Entwicklung ihrer Protagonistin, wobei meist nicht klar ist, ob dabei ,MARGARET ' oder ,NATASCHA' schreiben und reflektieren,

\footnotetext{
${ }^{23}$ Deutsche Ausgabe: Die Tagfalter Europas und Nordwestafrikas, übers. u. bearb. von WALter Forster, Hamburg, Berlin 1971.

${ }^{24}$ Gates, Barbara T. (1999): Kindred Nature: Victorian and Edwardian Women Embrace the Living World, University of Chikago Press.

${ }^{25}$ Ibid. S. 88 [„In Gebieten wie der Entomologie hatten Männer wie Riley sich angeeignet, was sich zu sehen gehörte, und deshalb war diese Abwertung von Fountaines Arbeit keineswegs nur vorgebracht mit einem Maß an Geringschätzung und Respektlosigkeit, sondern mit dem Glockenschlag der Autorität. Sein war das offizielle Wort, basierend auf der offiziellen Sichtweise; es war das, mit dem jeder von sich selbst überzeugte Wissenschaftler übereinstimmen würde. Autodidaktische Feldund Wiesenforscher waren einfach nichts wert und ungeeignet für die wissenschaftliche Unternehmung - und zum größten Teil landeten Frauen in diesem Feld der Autodidakten und Inoffiziellen."]

${ }^{26}$ Scott-Stokes, Natascha (2006): Wild and Fearless: The Life of Margaret Fountaine, London.

${ }^{27}$ Ibid S. 15 [, as an eccentric Victorian with an indiscriminate passion for men"].
}

weil die Anführungszeichen meist fehlen. Ihre professionellen Erfolge als Schmetterlingsjägerin treten dabei in den Hintergrund, die Entomologie wird auf den letzten Dutzend Seiten der Biographie abgehandelt. MARGareT FountaINe aber erscheint hier nicht zu Unrecht als Vorbild für die Selbstbefreiung der Frau und die Ermutigung, eigene Wege zu gehen.

Eine schöne Erinnerung produzierte am 15. April 2013 der Bayerische Rundfunk Radio Bayern 2 mit einem Kalenderblatt zum Datum 15. April 1878, dem Tag als Margaret Fountaine mit ihrem Tagebuch begann. Die Autorin UlRIKe RÜCKerT und die Sprecherin Ilse NeuBAUER stellen dabei das ,wunderbare Leben einer alten Jungfer' vor. Der Beitrag schließt mit dem schönen Satz: „Fünfzig Jahre lang war sie auf allen Kontinenten unterwegs gewesen, in ungefähr 60 Ländern. Ein ganzes Leben auf demselben kleinen Fleck in der großen Welt zu verbringen, hatte sie geschrieben, mache den Geist schwach und eng. ${ }^{\prime 2} 28$

Der ungarische Entomologe bairischer Herkunft Lajos Aigner-Abafi (Ludwig Aigner) benannte 1901 die weiße Form des Weibchens von Colias aurorina HerRICH-SCHÄFFER 1850 ssp. heldreichi STAUDINGER 1862 als f. fountainei.

Der britische Entomologe Arthur H. Bruce Rydon benannte 1971 eine Gattung neotropischer Nymphaliden als Fountainea, bekannteste Art: Fountainea nessus LATREILLE 1813.

\section{Literatur}

Higgins, L.; Riley N. D. 1970: A Field Guide to the Butterflies of Britain and Europe, London.

Higgins, L.; Riley N. D. 1971: Die Tagfalter Europas und Nordwestafrikas, Hamburg, Berlin. - Übers. u. bearb. WALTER Forster.

Fountaine, M. 1980: Love among the Butterflies, Times Newspaper Ltd, London Ed. W. F. CATER.

Fountaine, M. 1983: Ich sammle nicht nur Schmetteringe..., Reisen und Abenteuer einer viktorianischen Lady, Wien, Hamburg Hrsg. Von W. F. Cater (Berecht. Übers. aus d. Engl. Maria GridLING).

Fountaine, M. 1987: Butterflies and late Loves, The Further Travels and Adventures of a Victorian Lady, Ed. W. F. Cater, Boston.

Gates, B. T. 1999: Kindred Nature: Victorian and Edwardian Women Enbrace the Living World, Chikago.

Salmon, M. A. 2000: The Aurelian Legacy: British Butterflies and Their Collectors, Berkeley.

Sсотт-Sтокеs, N. 2006: Wild and Fearless: The Life of Margaret Fountaine, London.

\footnotetext{
${ }^{28}$ www.br.de/radio/bayern2/sendungen/kalenderblatt/1504margaret-fountaine-tagebuch-schmetterling-100html
} 